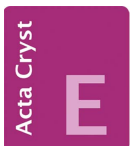

ISSN 2056-9890

\section{Bis(2-aminopyridine)dibenzoatocobalt(II). Erratum}

\author{
Wen-Zheng Ju, Rui-Hua Jiao, Ping Cao and Rui-Qin Fang*
}

Institute of Functional Biomolecules, State Key Laboratory of Pharmaceutical Biotechnology, Nanjing University, Nanjing 210093, People's Republic of China. *Correspondence e-mail: fangrq326@163.com

In the paper by Ju et al. [Acta Cryst. (2006), E62, m1012-m1013], the metal atom was reported incorrectly.

The authors of Ju et al. (2007) have indicated that the metal atom in the reported complex was assigned incorrectly. The paper is therefore withdrawn from the published literature.

\section{References}

Ju, W.-Z., Jiao, R.-H., Cao, P. \& Fang, R.-Q. (2006). Acta Cryst. E62, m1012-m1013. 